\title{
Chapter 12 Nanoplasmonic and Microfluidic Devices for Biological Sensing
}

\author{
G. Perozziello, A. Giugni, M. Allione, B. Torre, G. Das, M. L. Coluccio, \\ M. Marini, L. Tirinato, M. Moretti, T. Limongi, P. Candeloro, and \\ Enzo Di Fabrizio
}

\begin{abstract}
In this chapter we report about recent advances on the development and application of 2D and 3D plasmonic nanostructures used for sensing of biological samples by Raman spectroscopy at unprecedented resolution of analysis. Besides, we explain how the integration of these nanodevices in a microfluidic apparatus can simplify the analysis of biological samples. In the first part we introduce and motivate the convenience of using nanoplasmonic enhancers and Raman spectroscopy for biological sensing, describing the phenomena and the current approaches to fabricate nanoplasmonic structures. In the second part, we explain how specific multi-element devices produce the optimal enhancement of the Raman scattering. We report cases where biological sensing of DNA was performed at few molecules level with nanometer spatial resolutions. Finally, we show an example of microfluidic device integrating plasmonic nanodevices to sort and drive biological samples, like living cells, towards the optical probe in order to obtain optimal conditions of analysis.
\end{abstract}

\subsection{Introduction}

Plasmonic properties of metal nanoparticles have been exploited since long ago. For instance, artists have been using optical properties of metal nanodrops to generate brilliant colors, when shined by the direct sunlight, in glass artefacts and artwork for centuries.

G. Perozziello • M.L. Coluccio • P. Candeloro Bionem Lab, Departement of Experimental Clinics, Campus "Salvatore Venuta", University Magna Graecia, Viale Europa, 88100 Germaneto-Catanzaro, Italy

A. Giugni • M. Allione • B. Torre $\bullet$ G. Das $\bullet$ M. Marini $\bullet$ L. Tirinato $\bullet$ M. Moretti $\bullet$ T. Limongi $\bullet$

E. Di Fabrizio $(\bowtie)$

Physical Science and Engineering Division (PSE), King Abdullah University of Science and Technology (KAUST), Thuwal 23955-6900, Saudi Arabia

e-mail: Enzo.DiFabrizio@kaust.edu.sa 
When an electromagnetic field interacts with a metallic particle, among different possible processes, it can also excite a plasma oscillation, an electric-field-induced oscillation of the free electrons of the particle that strongly enhances the local electromagnetic field. The study and the control of the interaction between electromagnetic field and free charges is the topic of Plasmonics research. Plasmonics also investigates the mutual induction between conductive nanostructures through their electromagnetic fields in a classical and quantum framework, commonly applied to 2D and 3D metal nanostructures.

These electronic excited states can be classified, for example, as surface or bulk plasmons identified by their property to be confined or not at the interface between the metal and the dielectric. Each of them has a specific dispersion relation, very different the one of light. Energy and momentum conservation, in general, hinders their excitation by direct photons irradiation on the metal except for specific conditions and shape adopted, that define the optimal coupling geometries. That is why we commonly use nanostructured grating on a flat surface, or specific geometrical shapes and orientations of nanoparticles in respect to the incoming electromagnetic field. For scientific application, surface plasmons polaritons, SPPs, are the most fruitful ones; they are characterized by an evanescent electric field, fact that confines their actions tightly to the metal surface on the range of few tens of nanometers. For a single metal nanoparticle immersed in the electromagnetic field, the excitation of SPPs gives rise to a major spatial reshaping of the electromagnetic energy, generating intense subwavelength-localized spots at specific resonant frequencies. In clustered nanostructures, the enhancement of the electromagnetic field depends also by their interaction with each other. In general, the resonance also shift in response of a change in the dielectric constant of the medium around the nanoparticles, as it happens when metal nanoparticles merge with substances like water solutions or organic media.

In particular, for nanoparticles sensibly smaller than the incident wavelength, an efficient localized surface plasmon resonance (LSPR) occurs.

In this case, the external field $\boldsymbol{E}_{\boldsymbol{0}}$ can be approximated as uniform across each particle, and the electric dipole induced within the nanoparticle (Figs. 12.1 and 12.2a) generates an additional dipolar field $\boldsymbol{E}_{\text {dip }}$, so that:

$$
E_{t o t}=E_{0}+E_{d i p}
$$

By solving the electrostatic problem in the outer space of a spherical nanoparticle, as schematically represented in Fig. 12.2a, the electric potential comes out as the sum of a term due to $\boldsymbol{E}_{\boldsymbol{0}}$ and another one generated by a dipole moment $\boldsymbol{p}$ :

$$
\mathbf{p}=\pi \varepsilon_{0} \varepsilon_{d} a^{3}\left(\frac{\varepsilon_{m}-\varepsilon_{d}}{\varepsilon_{m}+2 \varepsilon_{d}}\right) \mathbf{E}_{\mathbf{0}}
$$

where $\varepsilon_{m}$ and $\varepsilon_{d}$ are respectively the dielectric constant of the metal and the dielectric surrounding medium, and $a$ is the radius of the nanoparticle. 


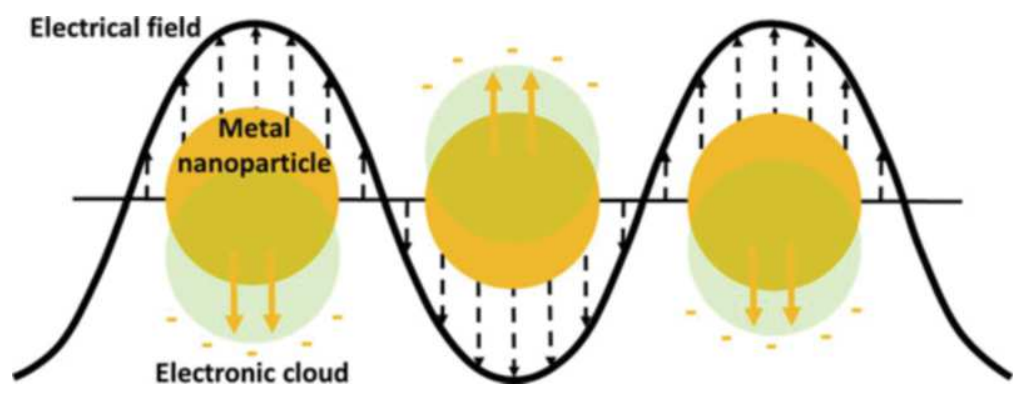

Fig. 12.1 Schematic representation of sinusoidal electric field acting on a metal nanoparticle

A

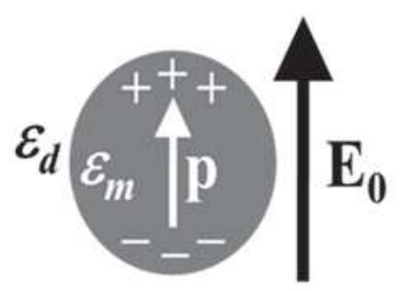

B

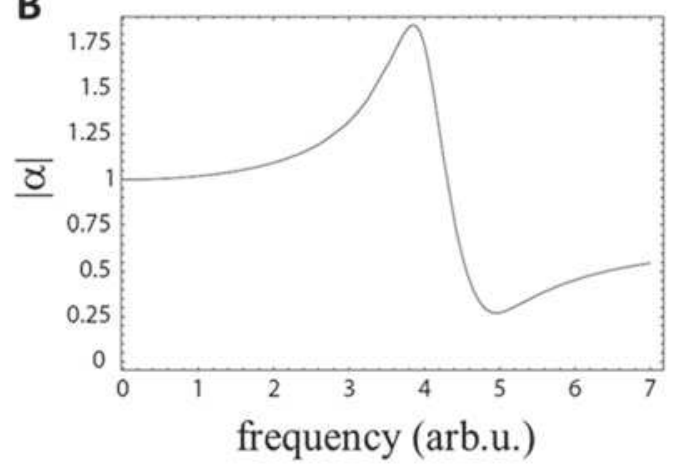

Fig. 12.2 (a) Schematic representation of a metal nanoparticle polarization under the effect of a uniform electrical field; (b) Graphical representation of the polarizability of that nanoparticle as a function of the electric field frequency

Introducing the electrical polarizability $\alpha$ and considering explicitly its frequency dependence, we can rewrite $\boldsymbol{p}$ as:

$$
\mathbf{p}(\omega)=\varepsilon_{0} \varepsilon_{d} \alpha(\omega) \mathbf{E}_{\mathbf{0}}(\omega)
$$

with:

$$
\alpha=4 \pi a^{3} \frac{\varepsilon_{m}(\omega)-\varepsilon_{d}}{\varepsilon_{m}(\omega)+2 \varepsilon_{d}}
$$

As shown in Fig. 12.2b, the polarizability $\alpha$ has a maximum when:

$$
\varepsilon_{m}(\omega)=-2 \varepsilon_{d}
$$


Without demonstrating it Ref. [1], the resonance condition occurs when:

$$
\omega=\frac{\omega_{p}}{\sqrt{1+2 \varepsilon_{d}}}
$$

where $\omega_{p}$ is the bulk plasmon frequency [1].

The resonance in the polarizability $\alpha$ leads to a resonance of the local electric field:

$$
\mathbf{E}_{d i p}=\frac{1}{4 \pi \varepsilon_{0} \varepsilon_{d}}\left[\frac{3(\mathbf{p} \cdot \mathbf{r}) \mathbf{r}}{a^{5}}-\frac{\mathbf{p}}{a^{3}}\right]
$$

An important application of LSPRs is in surface enhance Raman scattering (SERS) spectroscopy that takes place when a molecule is located on a metallic nanostructure or a roughened metal surface. At the base of the enhancement of Raman scattering is an increased electromagnetic near field due to excitations of LSPRs. Other contributions comes from more complex effects like a local increasing of optical density of states and a modified molecular Raman cross-section due to the changed environment, that contribute to increase the Raman excitation rate. Chemical effects also contribute to the final result. The highest SERS enhancement recorded to date is about $10^{14}$, achieved on roughened silver surfaces [1].

Raman spectroscopy is an optical technique that allows obtaining information on the vibrational spectrum of a sample. The combination of a Raman spectrometer with an optical microscope allows obtaining a chemical bonds map of the sample at a spatial resolution typically around $1 \mu \mathrm{m}$ (Fig. 12.3a). The main advantages of this technique are: (i) the capability to perform chemical analysis in a labelfree environment, for example in the case of a biological sample this reduces the steps necessary to pretreat the sample and avoids any spurious contribution due

A

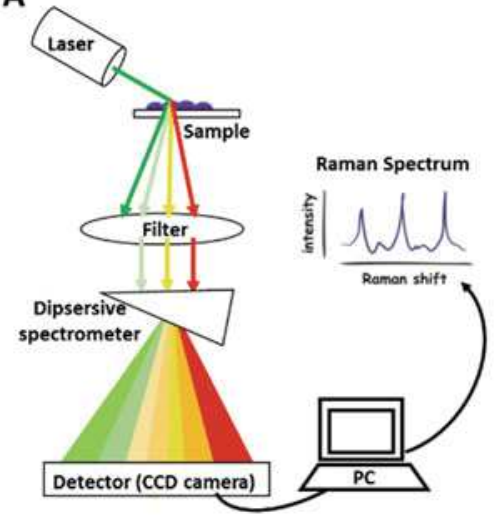

B

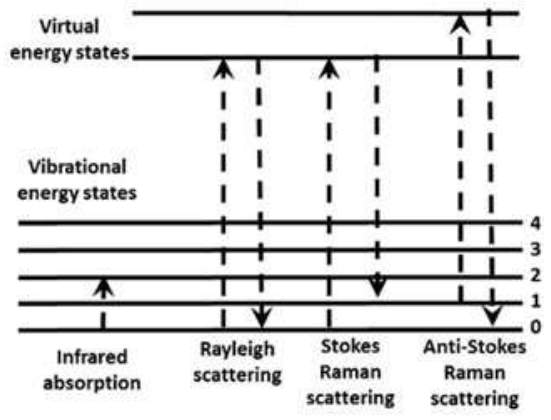

Fig. 12.3 (a) Schematic representation of the working principle of a Raman Spectroscopy Instrument (b) Scheme of energy levels of scattered light 
to introduction of fluorescent agents; (ii) the specificity of this analysis due to the unique fingerprint that each chemical has; (iii) the possibility to analyze biological substances in water/buffer solutions that support life conditions.

The vibrational spectrum is determined by the chemical composition of the sample. In particular, the Raman effect describes the inelastic scattering between a photon and a molecule mediated by its thermally populated vibrational or rotational modes. Due to the interaction, the energy of the incoming photon is shifted by the energy of probed mode. When the photon delivers its energy to the molecular vibration, the phenomenon is called Stokes scattering, while it is called Anti-Stokes scattering when it gains energy by de-excitation of a vibrational mode. This is schematically shown in the Fig. $12.3 \mathrm{~b}$ and more information can be found in Ref. [2].

Raman transitions can have very sharp lines enabling a detailed analysis of the molecule under study. Typically the Raman cross sections are more than ten orders of magnitude smaller than those of fluorescence processes $\left(\sigma_{\text {Raman }} \approx 10^{-30}\right.$ $\mathrm{cm}^{2} /$ molecule). This is the reason why it is fundamental to enhance the Raman signal through local surface plasmon resonance effects to detect few molecules. For molecules in proximity of metal nanoparticles (typically $\mathrm{Au}$ or $\mathrm{Ag}$ are the most commonly used metals), the Raman signal is strongly amplified by the coupled LSPR.

Plasmonics undergo a steadily increasing interest in Science for its countless applications in almost any research field in technology and life science. These studies are further motivated by the recent development of nano-fabrication technologies (electron beam lithography or ion beam milling) and of modern characterization techniques (as laser microscopy and scanning near field and electron microscopies).

Nowadays, advances in medicine mainly relies on new technical solutions and more sensitive methods to develop devices with very high sensitivity and selectivity.

When a biological sample, like the body fluids (blood, saliva, plasma, etc.) needs to be analyzed or screened, several challenges and issues need to be faced. These include the severe dilution of the biomolecules of interest, that are often well below the resolution limit of the most used screening procedures or the complexity of the same sample in terms of number of substances contained into it, which require a sample pretreatment allowing to resolve the analyses of a single particular substance. Nanoplasmonic devices offer realistic tools to face these problems. Artificial nano-devices can interact with biological objects (from cells, as large as ten micrometers, to biomolecules sizing few nanometers or less), and are, therefore, realistic candidates for solving these detection issues. The transition from the micron to the nanoscale dimensional control on surface features translates into increases in information quality, quantity and density, whereby the molecular detection, identification and diagnostics are significantly improved. In particular, nanoscale biophotonic devices, and especially SERS substrates, are capable to detect biological moieties in the limits of very low abundance ranges [3]. A large variety of plasmonic nano structures has been realized for applications in photonics, spectroscopic characterizations, biological sensing and 
detection of single molecules. These devices feature different designs, sometimes somehow exotic, including electrochemically modified electrodes, colloids, island films [4,5], adiabatic cones [6], or regular arrays of metallic nano-dots [7].

In the following sections we will show some example of plasmonic nanostructures used for biosensing. In particular we will show how nanospheres, aggregates of nanoparticles and nanostars can achieve SERS effect and could be used to analyze DNA at very low concentrations.

The last section of the chapter will explain how integrating nanoplasmonic structures in a microfluidic device it is possible to simplify biological analysis of single living cells. This sensor allows trapping single cells in proximity of the optical probe. This analysis strategy sorts single cell among a large population thus reducing acquisition time consistently.

\subsection{Novel Approaches for Optical Nanostructures Implementation}

\subsubsection{Plasmonic Self Similar Chains}

An electromagnetic field interacting with metallic nanoparticles induces a local field,, as a consequence of the plasma oscillation, as explained in the previous section. For example a single silver nanosphere can provide a field enhancement $\mathrm{g}=\mathrm{E} / \mathrm{E}_{0}$ around 30, a value that can be enormously increased in the case of several close nanoparticles, for the collective plasmonic oscillation in each nanoparticle. It was found that the highest values of field enhancement occur in particular points, called "hot spots", which fall in the gaps between metal nanoparticles [8]. On the basis of these observations the group of Mark I. Stockman had the idea to theoretically study self-similar systems composed of silver nanospheres, with the aim to create efficient nanolenses displaying hot spots with giant field enhancements [9]. These systems, designed and measured for application in Raman spectroscopy, offer specific active sites that can trigger giant Raman scattering from trapped molecules.

A plasmonic self-similar system may be composed of a chain of metal nanospheres. In this case, we indicate the radius of the $i$ th nanosphere by $R_{i}$ and its surface-to-surface separation, or gap, from the $(i+1)$ th nanosphere by $d_{i ; i+1}$. To obtain the self-similarity, the radiuses and gaps must obey to the relations:

$$
\begin{aligned}
& R_{i+1}=k R_{i} \\
& d_{i+1, i+2}=k d_{i, i+1}
\end{aligned}
$$

$k$ is a constant. 
a
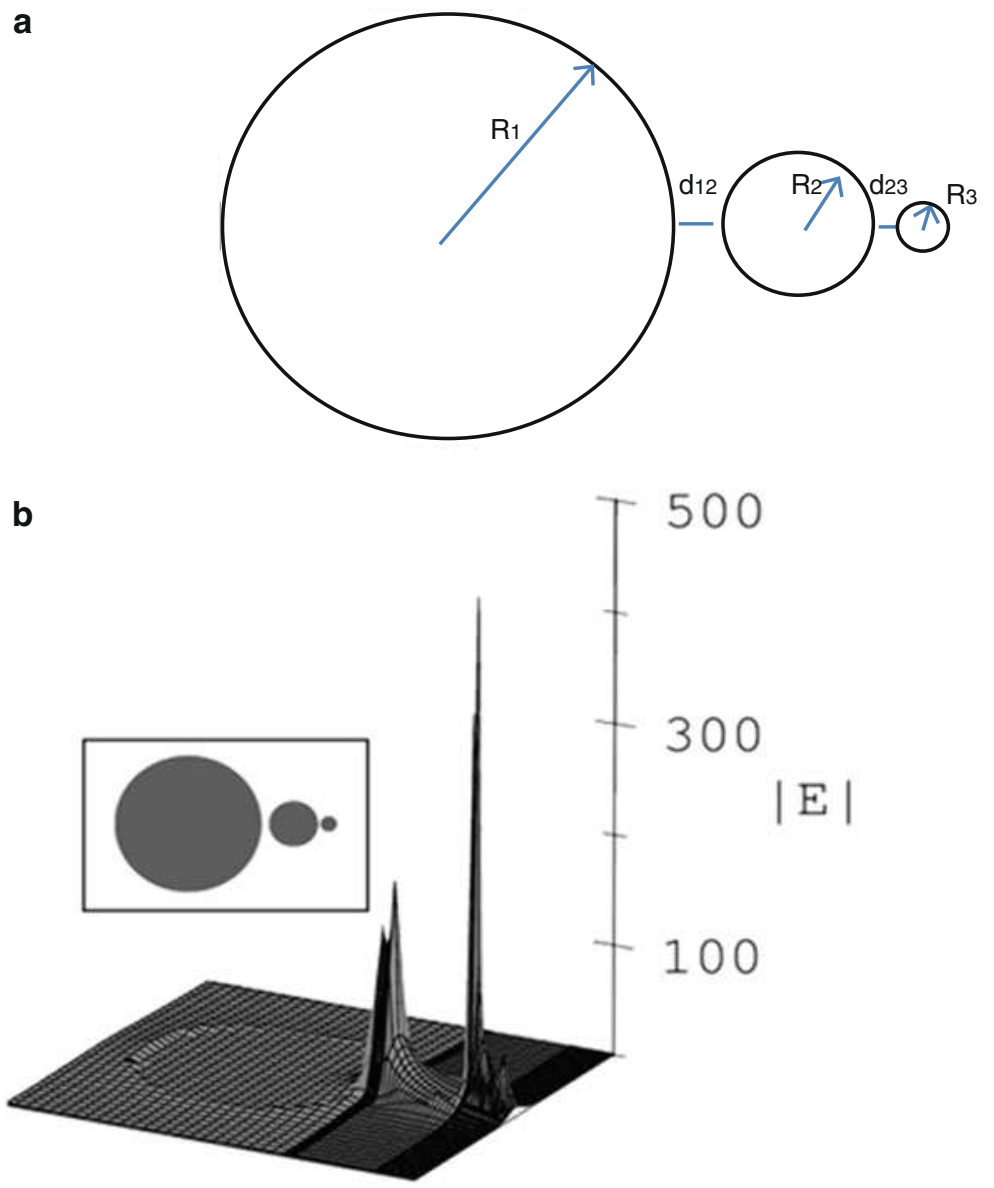

Fig. 12.4 (a) Schematic representation of a self-similar chain of three nanospheres. (b) Theoretical amplification of the electrical field in proximity of nanospheres in resonance conditions. The inset shows the cross section of the spherical nanoparticles used in the simulation, where the radius of the smallest nanosphere is $10 \mathrm{~nm}$. The values of the modulus of the electric field are normalized on the value of the electric field of the incident beam

In Fig. 12.4, it is represented a schematic drawing of a self-similar chain (SSC) consisting of three metallic nanospheres and a numerical simulation of the total electric field enhancement.

The efficacy of this system relies on the smallest gap, where a very large local field enhancement can be obtained. In fact, if Q is the resonance quality factor

$$
Q \approx \frac{\operatorname{Re} \varepsilon(\omega)}{\operatorname{Im} \varepsilon(\omega)}
$$


$\varepsilon(\omega)$ is the relative dielectric function of the metal and $\omega$ is the photon frequency, the local field $\mathrm{E}$ near the biggest nanosphere is enhanced by a factor $\mathrm{Q}$ with respect to the exciting field $\mathrm{E}_{0}$. It is noticeable that for a noble metal $\mathrm{Q} \gg 1$. The local field of this sphere excites the next nanosphere. For this second sphere, the excitation contribution from the exciting field $\mathrm{E}_{0}$ is negligible if compared to the excitation induced by the first bigger one because its polarizability, proportional to its volume, is much smaller (in this case $\mathrm{k} \ll 1$ ). The same phenomenon happens to the following couple of nanospheres, whose gap shows a field enhancement of $\mathrm{Q}^{2}$, and so on for the following nanospheres. Consequently the (n-1)th gap in this cascade produces a field enhancement of $\mathrm{Q}^{\mathrm{n}}$.

The great potentiality of a plasmonic SSC is clearly evident in Raman spectroscopy, where the cascade enhancement finds an optimal application in SERS. The SERS amplification factor $\mathrm{g}^{\mathrm{R}}$ goes approximately as the forth power of the local field enhancement $\mathrm{g}$ :

$$
g^{R} \cong g^{4}=\left(\frac{|E|}{\left|E_{0}\right|}\right)^{4}
$$

where $\mathrm{E}$ is the local optical electric field, and $\mathrm{E}_{0}$ is the electric field of the excitation wave [Ref]. In the case of $\mathrm{n}$ nanospheres, $\mathrm{g}$ can be expressed as:

$$
g(n)=\frac{E_{n}}{E_{0}} \approx Q\left(\frac{R_{n}}{R_{1}}\right)^{\frac{\ln Q}{\ln k}}
$$

A SSC of three nanospheres is already an optimal plasmonic nanolens. For example, in the specific case of nanospheres of silver for which Q 10, the smallest gap has an enhancement factor $Q^{3}=10^{3}$, corresponding to a Raman scattering enhancement of $10^{12}[10,11]$.

Experimentally a similar nanolens can be realized using a two steps process (Fig. 12.5). In the first step nanoholes which reproduce a SSC of spheres are drawn on a layer of electronic-resist spun onto a silicon wafer, by means of electron beam lithography. In the last step the nanoholes are filled of silver or gold nanoparticles by electroless deposition (ED).

$\mathrm{ED}$ is an autocatalytic redox reaction in which electrons are exchanged between metal ions in solution (here in particular silver or gold) and the substrate, which in this case is a silicon wafer. This specific substrate is particularly suitable for the ED technique due to the presence on its surface of dangling bonds, which act as deep-center defects, with a bound state that lies well below the conduction band [12]. The deposited metal nanoparticles size and density are controlled by parameters such as the concentration of the metal salt in solution (typically $\mathrm{AgNO}_{3}$ or $\mathrm{AuCl}_{3}$ ), the temperature and the time of the process. According to recent studies, the characteristic of lithographic pattern, in terms of absolute size and density of nanostructures in the pattern affect the process [13]. 


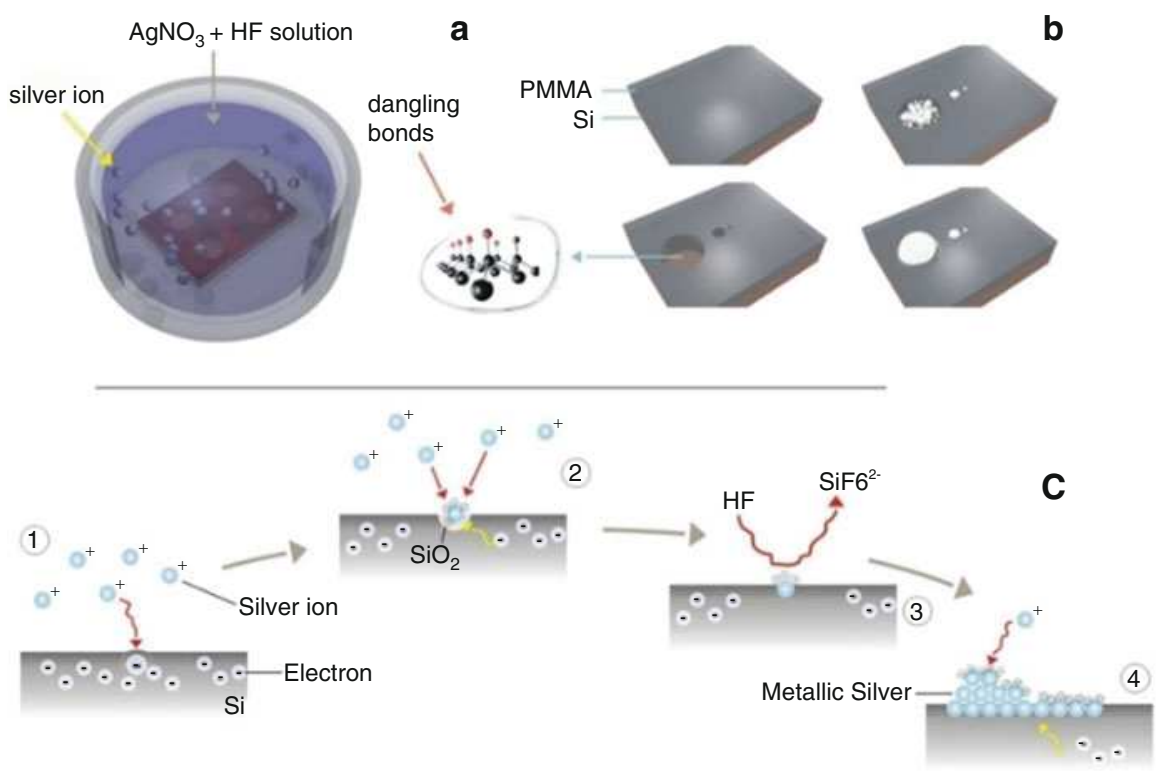

Fig. 12.5 Fabrication process of silver SSC. (a) After electron beam lithography and surface treatment with $2 \mathrm{M} \mathrm{HF}$, the sample is immersed in $\mathrm{HF} / \mathrm{AgNO}_{3}$ aqueous solution, where $\mathrm{Ag}+$ is reduced to silver metal through a redox reaction chain. (b) In nanowells (reduction surface), silver growth follows a spherical symmetry and generates three spheres of appropriate diameter and interdistance. (c) Redox reactions inside a nanowell starting from the silicon surface

Taking into account the previous considerations, silver SSC can be fabricated by immersion of the patterned silicon wafer in a solution of $\mathrm{HF} 0.15 \mathrm{M}$ and $\mathrm{AgNO}_{3}$ $0.05 \mathrm{mM}$ for $20 \mathrm{~s}$, at a temperature of $50{ }^{\circ} \mathrm{C}$ [14]. Into the patterned nanoholes the clusters of metal nanoparticles take a spherical shape, which in turn is advantageous for the plasmonic applications (Fig. 12.6). The method to create such devices ensures the reproducibility of the fabrication, the high controllability of position, size, and shape of the hot spots and the homogeneity in the field enhancement.

\subsubsection{3-D Nanostar Dimers}

Couples of 3D star-shaped gold nanostructures on silicon pillars (NOSP) represent another interesting kind of optical system. They can exploit the very high field enhancement that can be achieved when plasmonic nanostructures are tightly coupled.

Electron beam lithography (EBL) on a thick layer of poly(methyl methacrylate) (PMMA) spin-coated on a p-type Silicon wafer was used to define the shape of 
Fig. 12.6 SEM images of a silver SSC. The arrow indicates the smallest gap that is about $3 \mathrm{~nm}$

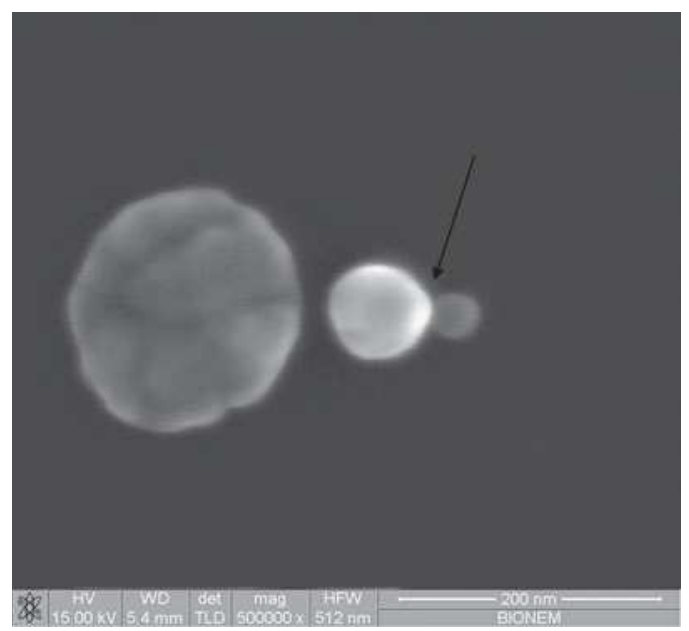

nanostars. This was followed by a deep reactive ion etching process (DRIE) to obtain the pillars, as explained in details in ref [Chirumamilla et al. Adv. Mater. 2014, 26, 2353-2358].

The star shape has the advantage with respect to other nanostructures of having multiple fine tips in the dimer and at the same time large inter-branches spaces important for the success of the DRIE process. Moreover, their complex design is reproducible with an inter-particle spacing (IPS) below $10 \mathrm{~nm}$.

The plasmonic behavior of these nanostructures depends from some parameters: the height of the dimer, the substrate material, and the IPS. In the cited paper, numerical simulations, before experimental tests, calculated electric field enhancement ( $E / E_{0}$, where $E$ and $E_{0}$ are the actual and initial electric fields) versus the dimer height, with the IPS constant. When dimers are directly in contact with Si bulk ( $h=0 \mathrm{~nm}$ ), low electric field enhancement is observed, due to the strong spread of the local electric field within the substrate; with the increasing of dimer height, the electric field enhancement increases too until the induced electric field in the dimers is completely decoupled from the substrate.

At small IPS $(<10 \mathrm{~nm})$, a strong mutual interaction of the surface plasmons of each nanostar causes a further localization and enhancement of the electromagnetic field in proximity of the IPS region (hot-spots).

These structures enable the detection of biomolecules at very low concentrations ( $1 \mathrm{pM})$, i.e. when there is only few or a single molecule per hot-spot. In Fig. 12.7a a sketch of the SERS working principle of a nanostars dimer is reported. Figure 12.7b, c show representative SEM images of the device and in Fig. 12.7d shows the SERS signal from a single or a few adenine molecules deposited on a NOSP device by chemisorption from a $1 \mathrm{pM}$ solution. 

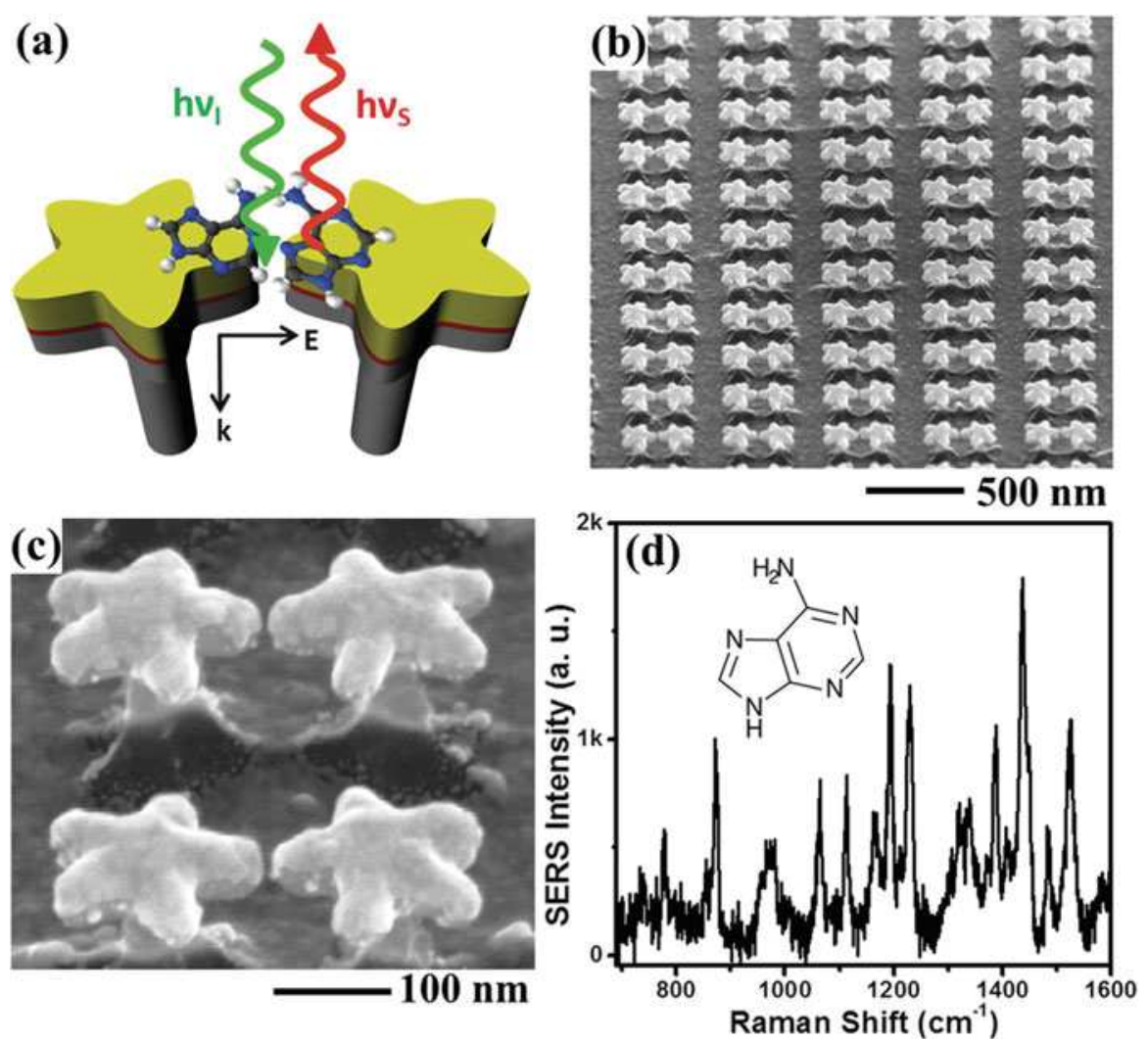

Fig. 12.7 (a) Illustration of the SERS working principle for a free-standing 3D nanostar dimers. $E$ and $k$ are the electric field and wave vector, respectively. $H v i$ and $h v s$ represent the incoming and scattered photon energy, respectively. (b, c) SEM images at a $52^{\circ}$ tilted view of the NOSP dimers with a 6-nm IPS and 150-nm Si pillar height. (d) Raman spectrum recorded from adenine that was chemisorbed from a solution at 1-p M concentration. The $\mathrm{k}$ in the intensity scale denotes 1000 . Inset: chemical structure of adenine. [14]

\subsubsection{Silver Decorated Nanotips}

Metallic cones can be used as plasmonic concentrators of electromagnetic energy. Their ability to focus and to amplify the optical radiation was studied by theoretical calculations and also experimentally demonstrated. In particular, silver and gold nano-cones can sustain adiabatic propagation and concentration at their apex of SPPs, with efficiency depending on their shape. Small apex angles provide optimal focusing and signal enhancement. This can be understood considering that SPPs coupled at the base of the cone from an incident far-field laser propagate 

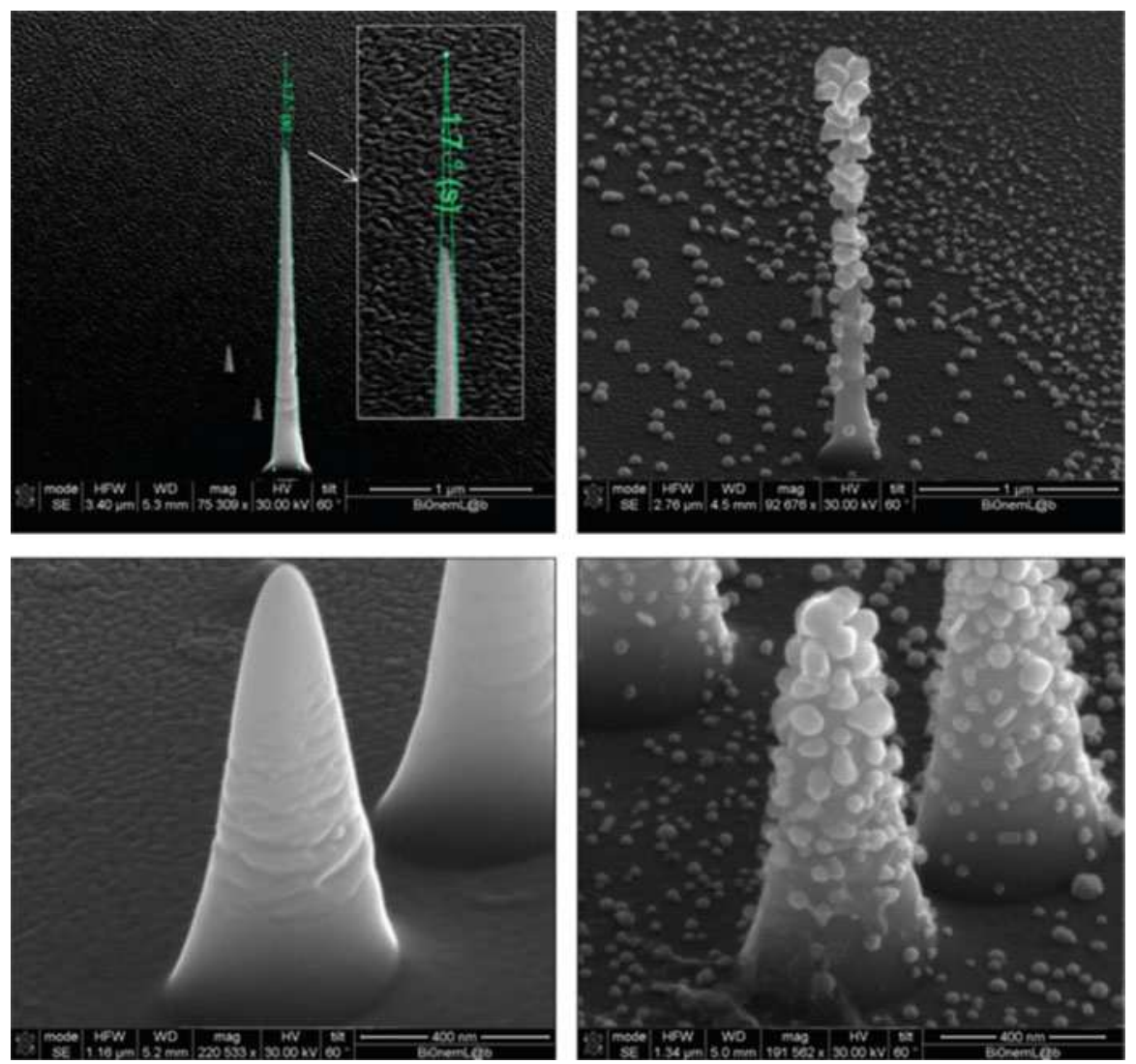

Fig. 12.8 SEM micrographs of cones. Cone before (left) and after silver electroless deposition (right), displayed here for two geometries: $\varphi=1.7^{\circ}$ (top) and $25^{\circ}$ (bottom), realized with a fixed silver salt concentration of $1 \mathrm{mM}$ [18]

towards the tip along the taper with decreasing wavelength and speed. As a result, SPPs accumulate at the tip of the cone providing a strong amplification of the electromagnetic fields.

The nano-cones shown in this section in Fig. 12.8, are realized by Electron BeamInduced Deposition (EBID), injecting into the chamber of a dual-beam system an organometallic platinum-carbon gas precursor $\left(\mathrm{CH}_{3}\right)_{3} \mathrm{Pt}\left(\mathrm{CpCH}_{3}\right)$ [15-17]. A pattern generator was used to adjust the parameters and consequently obtain different sizes of the cones.

Bare nanocones can be used for SERS applications, exploiting the field localization at the apex. By deposition on the cone surface of gold or silver nanoparticles, e.g. by electroless deposition, Raman signal emitted from molecules on the cones can be increased [18]. 
The peculiarity of this integrated system is the possibility to have a tridimensional probe in which the SERS efficiency may be adjusted simply varying the size and density of the silver nanoparticles from which the plasmonic behavior depends. At this purpose, it is possible to act on one hand on the concentration of the silver salt solution and on the other on the apex angle and height of the tip, since metal nanoparticles deposition is guided from the tip geometry itself. Silver nanoparticles in each case would preferentially distribute along the cone, with higher concentration at its apex (Fig. 12.8). For example, silver decorated tips has been used as SERS substrates for analysis of Rhodamine $6 \mathrm{G}$. That result suggests their implementation in microfluidic channels for analysis of complex mixtures of biological elements.

\subsection{SERS for Ultra-sensitive Detection of Biological Samples}

As described above, plasmonic nanostructures, in particular SSCs of silver nanospheres or dimers of metal nanostructures, allow the analyses of complex mixture or of molecules also in highly diluted conditions by Raman spectroscopy.

The strong plasmon localization, which is the peculiar characteristic of these nanostructures, produces a field enhancement ideal for SERS applications, so that their employment in Raman spectroscopy constitutes a powerful means to solve analytical problems with great sensitivity and specificity.

\subsubsection{Single Amino Acid Mutation Detection}

The micro-Raman spectrum of a biological sample is a complex mixture of signals coming from different macromolecules. The Raman bands originated from each component or molecular portion overlap each other, consequently the method is limited and not suitable for single-molecule or few-molecules detection so important in molecular medicine.

The advantage of silver SSCs with respect to another SERS device relies on the predetermined geometrical localization of the enhancement effect: a SERS device without this property amplifies Raman signal coming from all the substances deposited on it, without any spatial discrimination capability.

SSCs of silver nanospheres have been designed, fabricated and utilized as SERS substrate for early disease detection of breast cancer [19].

Coluccio et al. [19] adopted silver SSCs to detect biomarkers implicated in breast cancer development in human samples. Specifically they searched the single aminoacid mutation promoting cancer in a mixture of peptides. The BRCA1 protein is a tumor suppressor gene whose germline mutations predispose to breast and ovarian 
Fig. 12.9 SEM image at low magnification of a $\mathrm{m} \times \mathrm{n}$ matrix of nanolenses (SSCs). Each brigh spot in the figure is due to a single nanolens

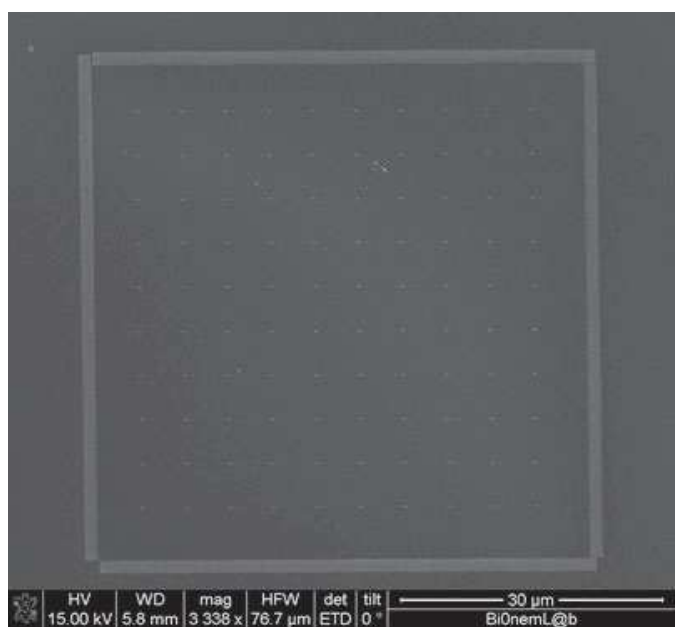

cancers [20]. The protein is functionally organized in domains located at the $\mathrm{N}$ terminus and $\mathrm{C}$ terminus. The $\mathrm{C}$ terminus has two amino-acid repeats, named $\mathrm{BRCT}$, having important roles in the stability of the BRCA1 protein conformation and in DNA transcription. This is the reason for which a mutation in the BRCT domain is an index of increased risk of developing breast and ovarian cancers. M1775R peptide is one of these missense mutations, where a methionine is substituted by an arginine at the fixed sequential position 1775 [21].

The direct detection of these single amino-acid mutations in the BRCA1 protein is impossible through Raman or other spectroscopic techniques because the signal of the mutation is overwhelmed by that of the whole protein.

$\mathrm{m} \times \mathrm{n}$ matrices of SSCs, with their high sensitivity, represent a good solution for this problem (Fig. 12.9).

The steps necessary for this kind of analysis by SSCs are: (i) preparation of the biological sample by breaking into mixtures of peptides the BRCA1 protein after the extraction from blood; (ii) deposition of each mixture on a SSCs array by drop-cast; (iii) drying of the samples in air; (iv) SERS measurement on each single nanolens of the matrix; (v) multivariate analysis.

At this purpose, Coluccio et al. utilized a matrix of $10 \times 10 \mathrm{SSCs}$, each made of three silver nanospheres, prepared by electron beam lithography and silver electroless deposition, as described before. Each single nanolens of the matrix is characterized by an intense hotspot localized in the smallest gap, whose enhancement ability depends mainly from the control of the dimension of the smallest nanosphere $(10 \mathrm{~nm})$ and the smallest gap itself $(<10 \mathrm{~nm})$. For obtaining good results with this detection system, the concentration of the biological sample should be accurately calibrated and the SSCs must be built with the proper dimensions. In fact, the concentration of the mixture determines the number of molecules that can fit into the volume of each hotspot: the average mutual distance of the molecules 
after deposition should be comparable with the linear size of the hotspot, in order that only few molecules can be found in the smallest gap after the solution has dried.

Regarding the choice of the matrix dimensions $(m \times n)$, a number of elements high enough to have all types of molecules statistically represented in the entire device is a fundamental condition to discriminate the components inside the mixture.

Exploiting the $10 \times 10$ matrix of SSCs, two fractions of around 12 peptides were analyzed by Raman spectroscopy in properly diluted solutions. One solution contained the M1775 peptide and the other the M1775R peptide.

At this point, detection of mutated peptides in BRCA1 becomes an analytical problem of finding the elemental composition of the multicomponent mixture. The full matrix is then measured, point by point, by a Raman spectrometer and the multicomponent spectrum at each position is fitted with a linear combination of the base elements, previously collected. A Raman spectrum, $\mathrm{s}_{\mathrm{M}}$, containing different peptids, can be fitted by the function:

$$
Y(f)=\sum_{i, j}^{n, m} x_{i, j} s_{i, j}(f)
$$

the index $i$ runs over the number of peptides $(i=1, \ldots, m)$, whereas $j$ runs through the number of intervals $(j=1, \ldots, n)$ in which spectra are divided. $\mathrm{m}$ is the wave vector range in the Raman spectra. The coefficient $x_{i, j}$ Are calculated by a least squares fit of $\mathrm{Y}$ with the experimental values $\mathrm{s}_{\mathrm{M}}$. By repeating the fitting procedure for all $m \times n$ matrix elements, all peptides extracted from BRCA1 are recognized [19].

This procedure ensures the possibility of detection of the mutated species in the mixtures. In Fig. 12.10b the Raman spectra of the peptide M1775 from pure samples, wild type and mutated, highlight the differences in the spectra between the two samples.

a)

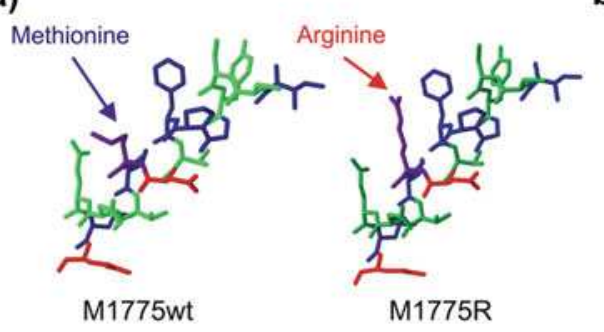

b)

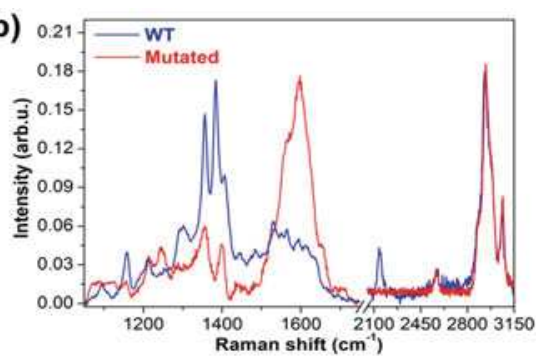

Fig. 12.10 Structure of wild-type and mutated peptides (a) and their Raman spectra (b) showing a clear difference between the spectra of the two peptides despite they are different by only the exchange of one amino acid (a methionine replaced by an arginine) 


\subsubsection{From Nucleotides to DNA Analysis}

Another interesting application of SSCs is the analysis of macromolecules and, in particular, the DNA sequencing. The working principle is the same as for the peptide mixture analysis.

The use of the SERS effect for oligonucleotides and DNA analysis could be attractive thanks to the efficiency of the technique and to its label-free character. Moreover, SERS can be combined with a microfluidic device to analyze oligonucleotides, label-free RNA or DNA in diluted solutions [22]. The advances in nanotechnology have allowed the development of such kind of devices, potentially very useful in medicine as instruments of diagnosis.

Recently an electrochemical surface-enhanced Raman spectroscopy aptasensor based on a functionalized silver nanoparticle was implemented for direct and specific detection of DNA hybridization for diagnosis in tuberculosis [23].

On the same line, the paper of Coluccio et al. [13] proposes the matrices of SSCs combined with data analysis to achieve label-free detection of DNA portions. Improving conventional SERS systems, it demonstrates the possibility to detect a single mutation or a very small fragment of nucleic acid by measuring changes in the spectrum that would be undetectable using conventional Raman.

The implemented device is still a matrix of $\mathrm{m} \times \mathrm{n}$ SSCs. The measurement was performed collecting the signal from any individual SSC generated by the fraction of nucleotides drop-cast on them.

The authors report the analysis of 6 and 9 bases oligonucleotides, which differ only for few bases. For each one, the most relevant spectra were compared to each other and to the spectra of isolated DNA bases and then fitted using a linear combination of the nucleobases. An example of the obtained spectra and of the array used is reported in figure (Fig. 12.11).

The SERS spectra of the oligonucleotide reported in figure exhibit few common bands at which other vibrational bands are superimposed, with differences that are attributed to the different orientations of the adsorbed molecules on the surface. The spectra were fitted to the expression in Eq. 12.12.

This led, in the case of a nucleotide with 9 bases (CCC GCC GCT), to a calculated relative content of the bases that resulted in very good agreement with the known real values, as can be seen by the following comparison

$$
\begin{aligned}
& A=0.044 \\
& C=0.565 \\
& G=0.2256 \\
& T=0.161
\end{aligned}
$$

These values are comparable to the theoretical, which are:

$$
\begin{aligned}
& \mathrm{A}=0 \\
& \mathrm{C}=0.6667 \\
& \mathrm{G}=0.2223 \\
& \mathrm{~T}=0.1111 .
\end{aligned}
$$



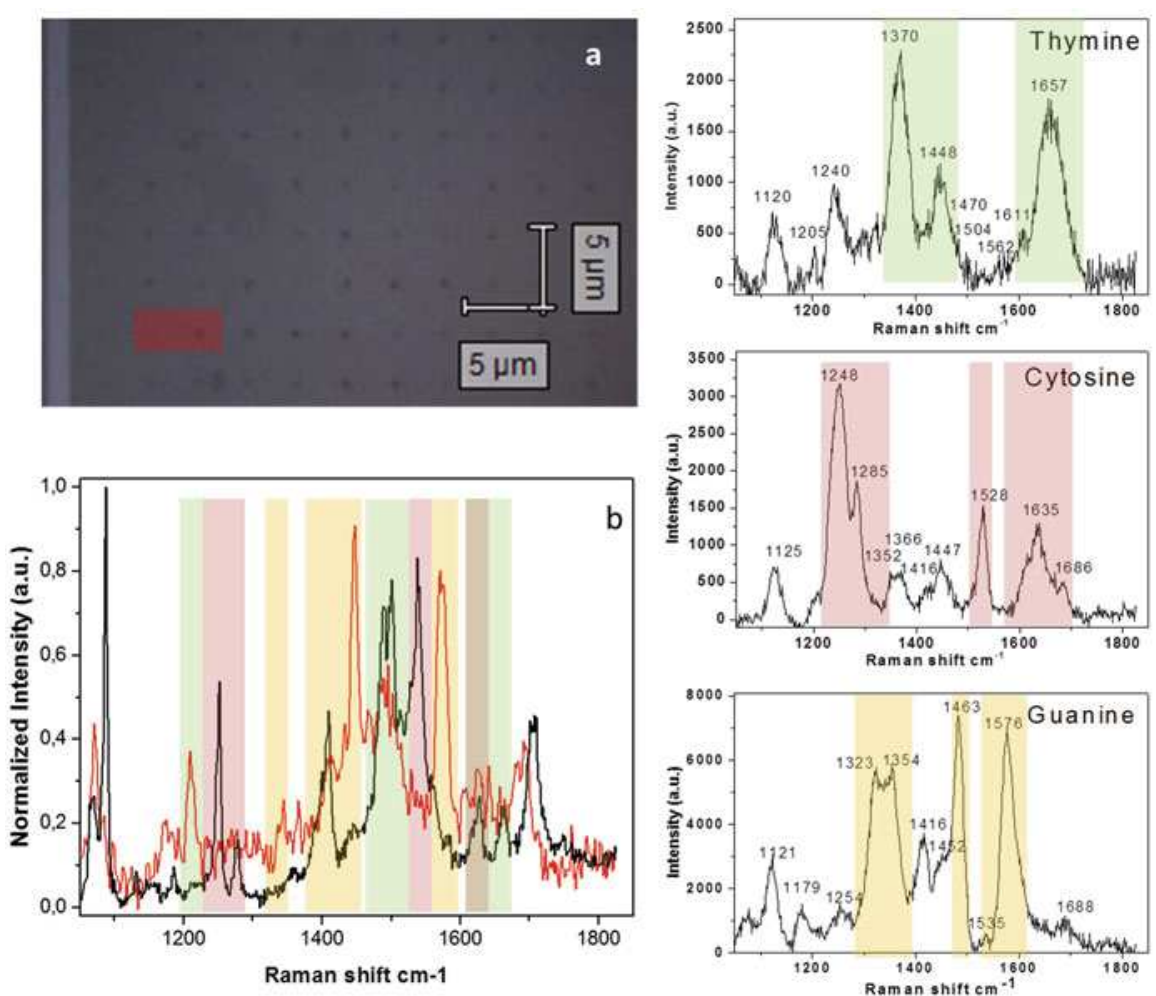

Fig. 12.11 Oligonucleotide p9 (CCCGTT): (a) optical image of the device. The red rectangle highlights the Raman mapped area around two nanolenses; (b) SERS spectra obtained in two hot spots of the Raman mapping compared with SERS spectra of the three DNA bases. The colored bands evidence some of the principal peaks of the three nucleotides [13]

The method just described can be applied in all cases in which the dimension of the nucleotide is less than $3 \mathrm{~nm}$ and thus two or three molecules at most fall into the hot spot of each nanolens. Figure 12.12 sketches how the Raman signal only comes from the hot spot of a nanolens discriminating the molecules, or the portions of a molecule, held in the area where the strong localization of the electromagnetic field is confined.

In the case of DNA analysis, the molecule is much longer than oligonucleotides. Since the hot spot for field enhancement in one of our SSCs has a dimension that is limited by the value of the last gap to $10 \mathrm{~nm}$, which also poses a limit on the maximum extension of the hot spot in all directions, it turns out that the maximum number of basis that can be probed at the same time is about 30 , being single nucleotide $0.33 \mathrm{~nm}$ in length. As a consequence, DNA or oligonucleotides with a larger number of bases will give very different spectra depending on their specific portion that falls into the hot spot (Fig. 12.13).

This SSCs, coupled with a device for realizing strand and scrolling of a DNA filament, could become a relevant technique for DNA sequencing. 


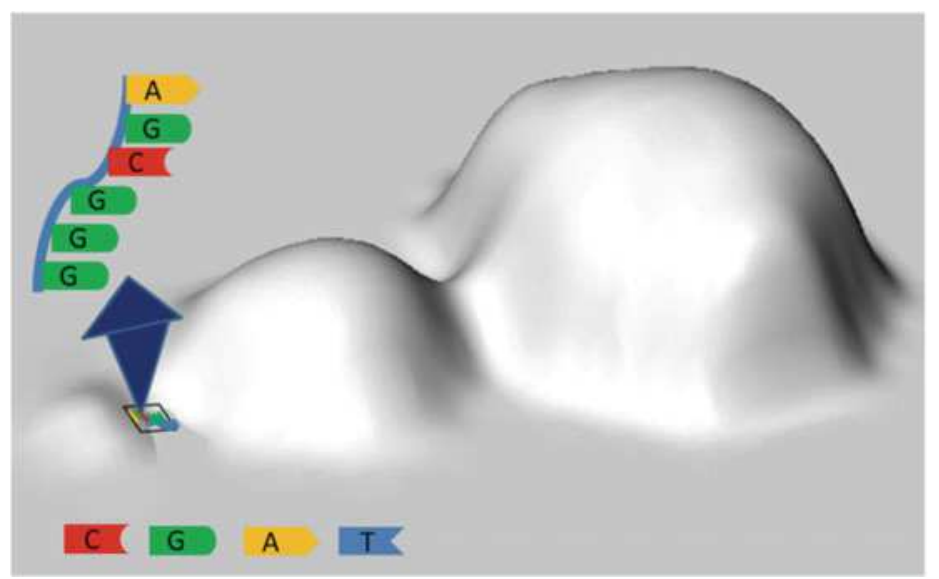

Fig. 12.12 Schematic representation of a Raman analysis on a layer of biomolecule in the smallest gap of a SSC: the Raman signal from the smallest gap dominates the intensity spectra

גDNA - Primer 1: M7-A1843P

GCT ATG GGC CCT TCA CCA ACA GGC CCA CAG ATC

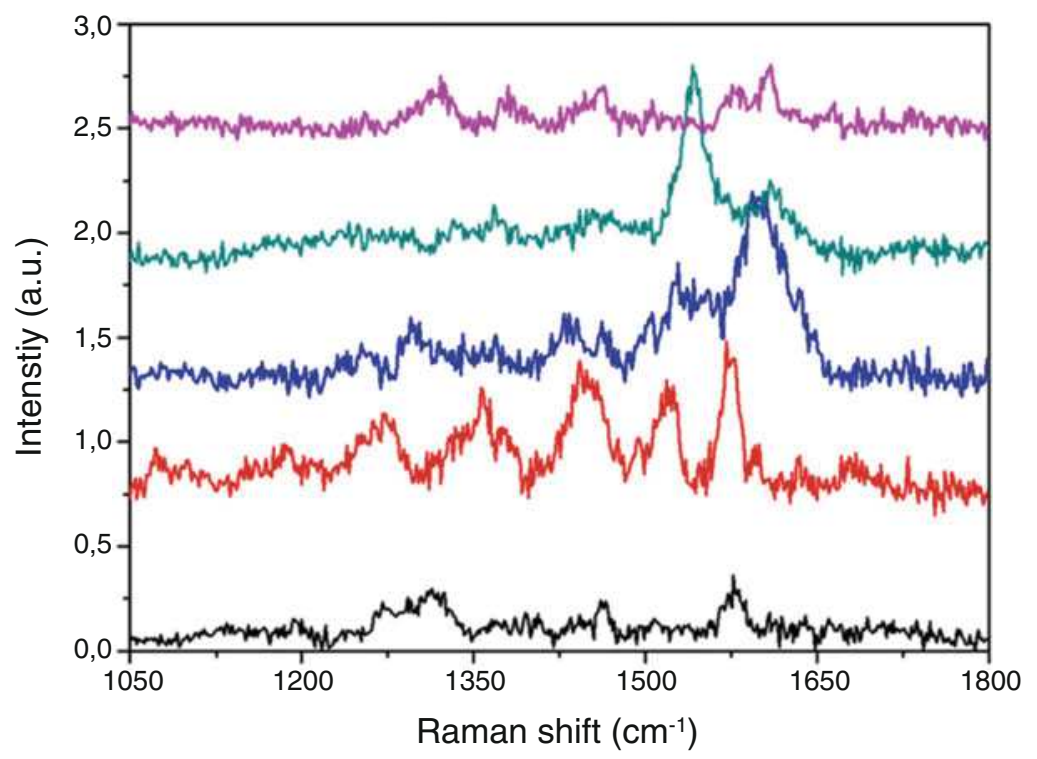

Fig. 12.13 Spectra of 33 bases portions of $\lambda$ DNA measured on various nanolenses 


\subsection{Microfluidic Devices Integrating Plasmonic Nanostructures for Biological Sensing}

\subsubsection{Raman Spectroscopy Compatible Microfluidic Devices for Cell Analysis}

Microfluidics focuses on the transport, manipulation and analysis of small amount of liquids, cells and particles. Microfluidic devices have been developed and exploited from several research groups as bioanalytical tools to give several advantages over conventional bioanalytical technologies. In fact, these devices guarantee high portability, simplified pretreatment protocols, accurate handling and low consumption of samples and reagents, as well as high resolution analysis and low production costs [24].

There are many examples in literature of microfluidic devices used for analytical purposes [25, 26]. These have been developed for DNA detection [27-29], protein analysis [30-32] and biomarkers [33, 34], discriminating healthy from tumor cells $[35,36]$, phenotypes screening [37, 38], and cells mechanical stress and deformability investigations [39-41].

Among these applications, a microfluidic device capable of analyzing the composition of a single cell at a subcellular level would push a step forward the conventional analysis on cells.

It is well known that individual cells, even those identical in appearance, differ in many characteristics. Due to this heterogeneity, traditional biochemical assays, which analyze cells in bulk, do not allow to get specific information at single cell level allowing investigating their activities or testing possible new biomarkers. Microfluidics allows a sequential handling and manipulation of cells which is significant for scientific research and clinical diagnostic applications.

Commonly, monitoring of cells over a long period of time is most frequently performed by fluorescence microscopy. However, it is quite common that the fluorescent dyes used for specific subcellular staining interfere with the development of cells, changing their phenotype and metabolism. Another limitation is that fluorescent dyes bleach quite fast. Finally, this technique only allows investigating molecules already known. These aspects make fluorescence staining strategies less desirable with respect to label free sensing.

This can be done using Raman Spectroscopy combined with plasmonic nanostructures integrated into microfluidic devices.

Raman spectroscopy has been largely used to determine cell conditions, such as living cells [42], dead cells [43], apoptotic cells [44], proliferating cells [45], and differentiating cells [46]. Raman spectroscopy can be integrated in microfluidic devices to achieve an accurate monitoring of single cells, allowing them to stay over long period in physiological or controlled environments [47-49].

Finally, the integration of plasmonic nanodevices in the microfluidic tool allow increasing significantly the resolution of analysis. 
Raman spectra are only minimally affected by the presence of water. This peculiarity is crucial when dealing with measurements on living cells that are always immersed in an aqueous buffer.

On the other hand, when microfluidic devices are combined with Raman Spectroscopy it is necessary to select only materials that give negligible contribution to the Raman spectra. This constrains leads to exclude all the polymers which give a strong Raman signal in the characteristic spectral range of cells, being polymers made by carbon chains, similarly to biomaterials. The concurrent strategies are: (i) use materials which give different Raman bands from the biological species or negligible signal amplitude; (ii) design a proper geometrical configuration to reduce the background, like for instance using metal shields and working in reflection [39];

In the following section, we show an example of a microfluidic device used to take measurements by Raman Spectroscopy on living cells [50]. The device is based on the use of a microfluidic trap integrated with a plasmonic Raman enhancer. It is composed by two slides of calcium fluoride, giving a negligible Raman signal, between which a photosensitive resist is placed. Five through-channels, integrating a microfluidic trap, are fabricated in the resist by photolithography, connected to external tubes by means of fluidic gaskets and a mechanical holder. A picture of the final device is shown in Fig. 12.14.

The trap is realized by means of a simple volumetric constriction of the channels (Fig. 12.15a).

Cells flowing in the microchannel can be blocked at each trap where a Raman spectrum can be collected with a minimum acquisition time of few seconds. The

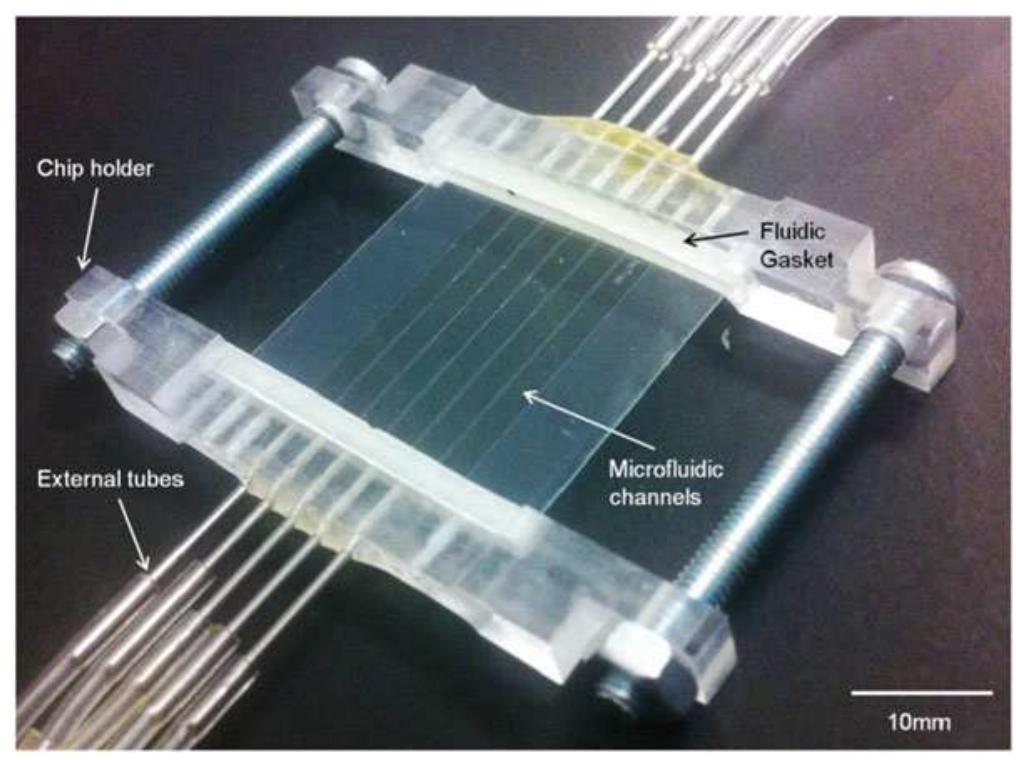

Fig. 12.14 Microfluidic device for Raman Spectroscopy on living cells 


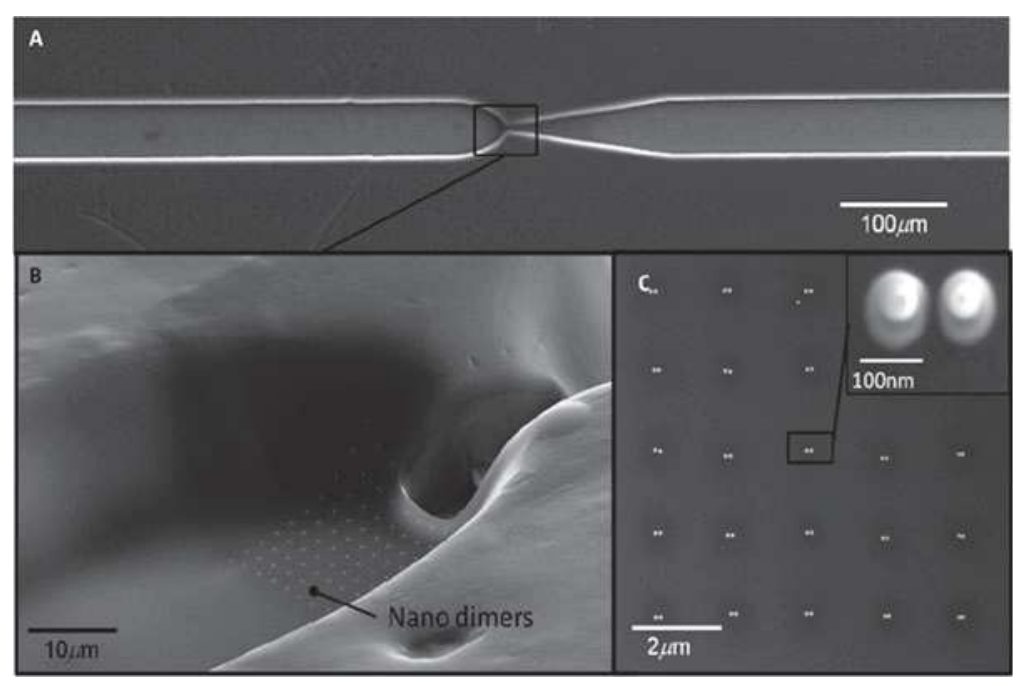

Fig. 12.15 Scanning electron image of the microfluidic trap and the integrated array of nanodimers. (a): Top view; (b): isometric view; (c): zoom in the area where the nanodimers are integrated

traps integrate plasmonic nanodimers (Fig. 12.15b, c). These plasmonic nanodevices enhancing Raman scattering increase the sensitivity and enrich the information about the cellular membrane and the cytoplasm of the trapped cell.

In principle, this geometry can be tailored for every kind of cell simply adjusting the size of the channels and of the traps.

These devices are designed to control a temporarily trapping by dynamically changing the flow conditions. When a constant flow rate is applied, a cell is moved toward the trap where it stops. Here the fluidic resistance and the pressure drop across the cell increase locally. The resulting force acting on the cell causes its deformation and pushes it through the trap (Fig. 12.16). After that, another cell can approach the trap. The controlled flow determines the cell transit time, approximately of $3 \mathrm{~s}$, enough to record a Raman spectrum. In such a way, it is possible to perform a continuous analysis of cells by Raman Spectroscopy in a passive way.

Such a device can be used in different ways: (i) the same cell can be moved forth and back in the trap by changing direction of the flow so that measurements on different portion of the same cell can be taken [Perozziello et al. microelectronic Engineering]; (ii) one single measurement on a cell sitting on the nanodimers in the field of view can be taken, getting information from one single cell membrane. The measurements from single cells of the same type can be also integrated together; (iii) micro-Raman by using objectives with higher NA can be taken, in this case the measurement can be used to search for specific information about the cell membrane and cytoplasm. 


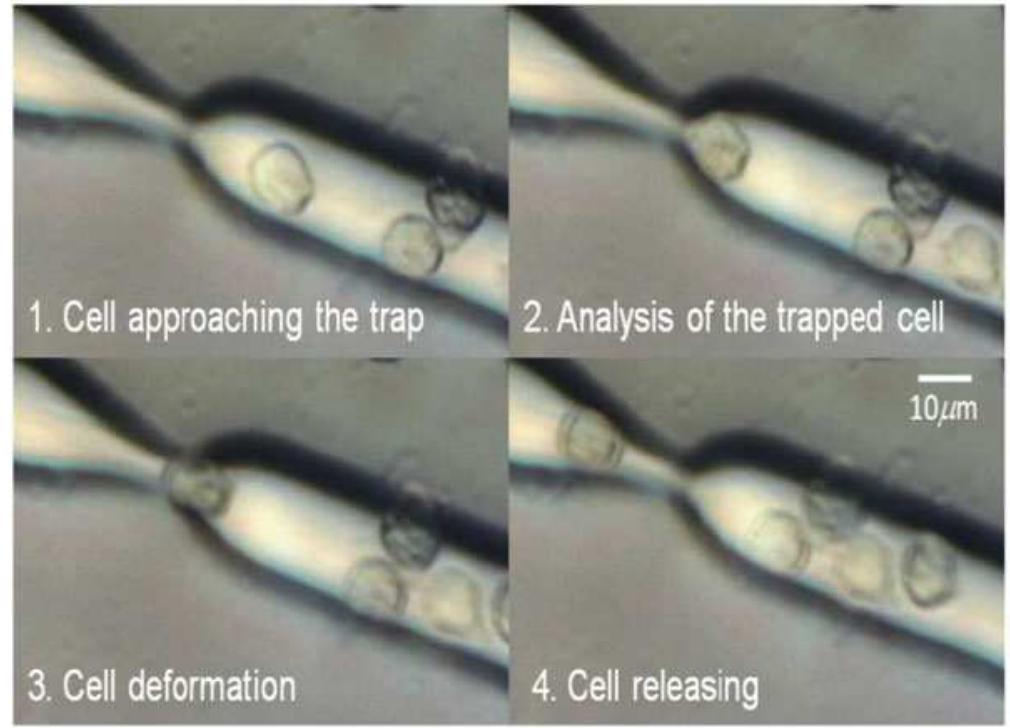

Fig. 12.16 Sequence of the cell analysis mechanism: (1) the cell approaches the trap followed by other cells ( $(t=0 \mathrm{~s})$; (2) the cell is trapped and the flow is stopped ( $t=2 \mathrm{~s})$, in this phase Raman measurements can be performed on the cell $(t=4 \mathrm{~s})$; (3) the increasing pressure due to clogged channel deform the cell forcing it through the trap $(t=6 \mathrm{~s})$; (4) the cell is released continuing to move over the trap while other cells are approaching the trap aligning themselves to it $(t=8 \mathrm{~s})$

The microfluidic devices were fabricated on calcium fluoride $\left(\mathrm{CaF}_{2}\right)$ slides on which a laminated dry photoresist was attached. The microchannels were defined by means of UV-photolithography. Subsequently, the plasmonic nanodevices were fabricated on top of the $\mathrm{CaF}_{2}$ substrate (Fig. 12.15b, c). These consist in a matrix of dimers of $100 \mathrm{~nm}$ diameter gold nanodots, separated by a gap of $10 \mathrm{~nm}$. These were fabricated using a FEI Nova Nanolab 600 dual beam focused ion beam system introducing an organometallic gas precursor $(\mathrm{CH} 3) 3 \mathrm{Pt}(\mathrm{CpCH} 3)$ to produce the $\mathrm{PtC}$ alloy. The electron acceleration was set at $30 \mathrm{keV}$ and the current at $0.15 \mathrm{nA}$. The resulting matrix, covering a square area of $20 \mu \mathrm{m}$ in size with a $2 \mu \mathrm{m}$ pitch, was then sputtered with $20 \mathrm{~nm}$ of gold and ion beam milled [50]. Subsequently, another slide of calcium fluoride was bonded using a hot press on top, to seal the micro channels.

\subsubsection{Single Living Cells Study}

The instrument used to perform Raman spectroscopy on the cells was a Renishaw inVia Raman Microscope mounting a 50X objective. Syringe pumps were connected to the microfluidic devices by Teflon tubes ( $0.4 \mathrm{~mm}$ ID -Internal Diameter), loaded with the biological samples. Such set-up was used to inject $1 \mathrm{ml}$ of biological 


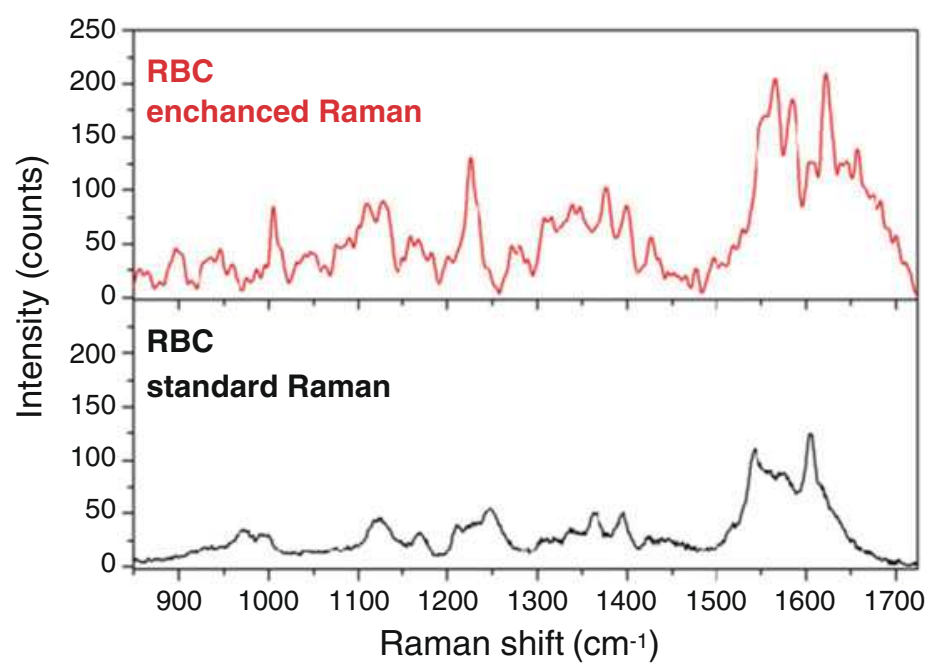

Fig. 12.17 Raman spectra collected from red blood cells (RBCs) on nanodimers (used power for the enhanced Raman measurements is $10 \%$ of the total) and on a flat substrate (used power for the standard Raman measurements is $100 \%$ of the total). The spectrum recorder on nanodimers exhibits a stronger signal and with higher detail spatial mapping

sample containing cells diluted at a concentration of about 5000 cells $/ \mu 1$ in PBS. The flow rate was set at $5 \mu \mathrm{l} / \mathrm{min}$ so that the cells could proceed very slowly and be stopped in correspondence of the trap. For the measurements, a laser with a nearinfrared wavelength $(832 \mathrm{~nm})$ was used. The power used to analyze the sample was $10 \mathrm{~mW}$ and the signal collection time was $3 \mathrm{~s}$. During Raman measurements the objective was focused at the level of the dimer gap in order to fully exploit the signal enhancement.

The large enhancement of the electromagnetic field at the gap of the dimers produces an enhancement of the Raman signal of biomolecules, as proved comparing the measurements taken on red blood cells trapped on top of the nanodimers and on a flat surface (Fig. 12.17). While the measurements taken on a flat substrate needed a laser power of a $100 \mathrm{~mW}$ to record a spectrum, only a power of $10 \mathrm{~mW}$ was needed to obtain a comparable signal on nanodimers. This is a very important point to consider when dealing with living biological samples since a high laser power can affect the viability of the cells.

The SERS spectrum showed not only a higher signal, compared to standard Raman, but above all the curve exhibits a richer peak profile due to plasmonenhancement of biomolecular vibrations occurring at the dimers locations.

Figure 12.18 shows Raman spectra collected over red blood cells (RBCs), peripheral blood lymphocytes (PBLs) and K562 tumor cells from leukemia in correspondence of the trap. Each spectrum is the average of the signals recorded from ten cells of the same type to provide a typical spectrum for each specific type. We stress that each SERS spectrum brings information from a very localized part 


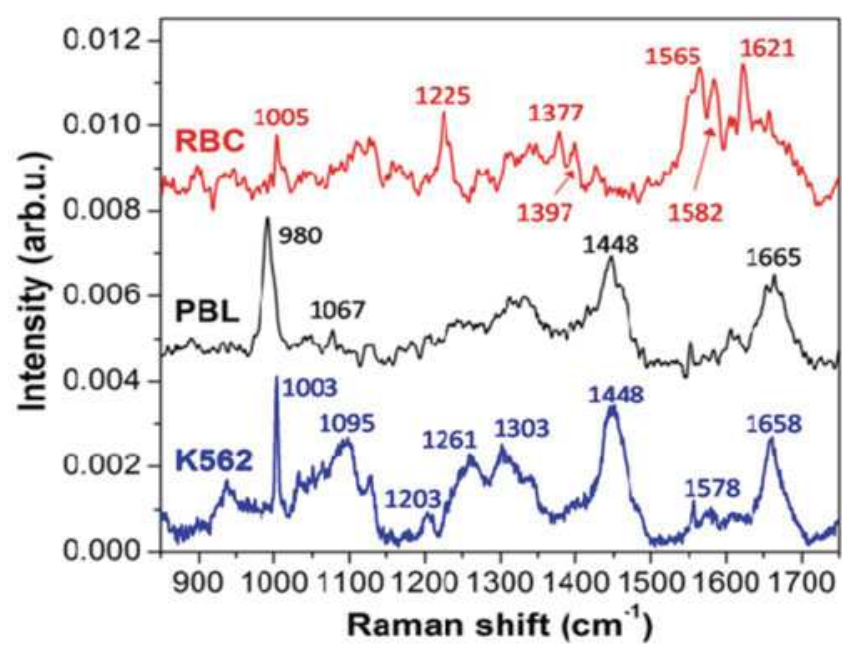

Fig. 12.18 Raman spectra collected in three different experiments with different cell lines, in the proximity of the microfluidic traps. From top to bottom average spectra of red blood cells (RBC), peripheral blood lymphocytes (PBL), and K562 tumor cells from leukemia are reported

of the cell and in particular from its membrane. In standard Raman spectroscopy, even using a high NA objective, the measurements on cells is an average of this information with that from the cell cytoplasm.

Concerning the red blood cells, it is possible to observe the typical Raman peaks of phenylalanine at $1005 \mathrm{~cm}^{-1}$, the deformation of the $\mathrm{C}_{\mathrm{m}}-\mathrm{H}$ bond at $1225 \mathrm{~cm}^{-1}$, the half- and quarter-ring stretching of pyrimidine respectively at 1377 and $1397 \mathrm{~cm}^{-1}$, and finally several peaks of the multi-structured band between 1540 and $1630 \mathrm{~cm}^{-1}$. The oxygenated state of RBCs can be deduced by the relative intensities of the $1565 \mathrm{~cm}^{-1}$ peak of the $C \beta C \beta$ stretching, the one at $1582 \mathrm{~cm}^{-1}$ of $\mathrm{C} \alpha \mathrm{Cm}$ asymmetric stretching, and the $1621 \mathrm{~cm}^{-1}$ peak of $\mathrm{Ca}=\mathrm{Cb}$ stretching, as already reported in literature [51],

The signature of PBLs is evidenced by the deoxyribose vibrations, at 980 and $1448 \mathrm{~cm}^{-1}$ [52], the phospholipid C-C stretching at $1067 \mathrm{~cm}^{-1}$, and the Amide I vibration from proteins secondary structure at $1665 \mathrm{~cm}^{-1}$. The broadening of $1448 \mathrm{~cm}^{-1}$ peak is due to the overlap of deoxyribose signature with $\mathrm{C}-\mathrm{H}$ deformation from protein.

Finally, the last curve shows the Raman signal from K562 cells. Comparison with literature shows a very good agreement: the phenylalanine ring mode is at $1003 \mathrm{~cm}^{-1}$ [53, 54]; the PO2- backbone vibration arises at $1095 \mathrm{~cm}^{-1}$; Amide III vibrations from proteins appear at 1203 and $1303 \mathrm{~cm}^{-1}$, where the former one overlaps with DNA bases signal; C-H bending and deformation are found at 1261 and $1448 \mathrm{~cm}^{-1}$, respectively. A small DNA signal is also observed at $1578 \mathrm{~cm}^{-1}$, and finally the Amide I strong vibration from proteins is located at $1658 \mathrm{~cm}^{-1}$. Compared to PBL, the Amide I shift towards lower frequencies (from 1665 to 
$1658 \mathrm{~cm}^{-1}$ ) is likely due to a larger presence of alpha helix structure of proteins. This is also supported by the small peak observed at $935 \mathrm{~cm}^{-1}$ (alpha helix C-C skeletal mode) for the K562 curve.

\subsection{Conclusions}

In this paper we collected part of our activity on nanoplasmonics for sensing and showed the application in biosensing. It is clearly appearing that the combination of plasmonics, microfluidics and spectroscopy is an interesting strategy for improving the detection sensitivity, specificity and complexity of mixtures.

The combination with microfluidics opens the possibility to extend the detection to single molecule but increasing the throughput analysis. It is our belief that this approach will become of commercial interest and medicine with accurate and personalized diagnosis will benefit from this approach.

Acknowledgments We acknowledge all contributors that contributed in this work from other institution, in particular the researchers from the previous Nanostructure Division at IIT (Italian Institute of Technology) in Genoa.

We thank for the financial support the King Abdullah University of Science and Technology start-up fund and the Italian Minister of Health (projects nos. GR-2010-2320665 and GR-20102311677)

\section{References}

1. Maier, S. A. (2007). Plasmonics: Fundamentals and applications. New York: Springer. ISBN 0-387-33150-6.

2. Giugni, A., Torre, B., Allione, M., Gentile, F., Candeloro, P., Coluccio, M. L., Perozziello, G., Limongi, T., Marini, M., Raimondo, R., Tirinato, L., Francardi, M., Das, G., Proietti Zaccaria, R., Falqui, A., \& Di Fabrizio, E. (2015). Novel plasmonic probes and smart superhydrophobic devices, New tools for forthcoming spectroscopies at the nanoscale. NATO Science for Peace and Security Series B: Physics and Biophysics, 68, 209-235.

3. De Angelis, F., Gentile, F., Mecarini, F., Das, G., Moretti, M., Candeloro, P., et al. (2011). Breaking the diffusion limit with super-hydrophobic delivery of molecules to plasmonic nanofocusing SERS structures. Nat Photonics, 5, 682-7.

4. Le Ru, E. C., Etchegoin, P. G., Grand, J., Felidj, N., Aubardì, J., \& Lévi, G. (2008). Surface enhanced Raman spectroscopy on nanolithography-prepared substrates. Current Applied Physics, 8, 467-70.

5. Qiu, T., \& Chu, P. K. (2008). Self-selective electroless plating: An approach for fabrication of functional 1D nanomaterials. Materials Science and Engineering, 61, 59-77.

6. De Angelis, F., Das, G., Candeloro, P., Patrini, M., Galli, M., Bek, A., et al. (2010). Nanoscale chemical mapping using three-dimensional adiabatic compression of surface plasmon polariton. Nature Nanotechnology, 5, 67-72.

7. Das, G., Mecarini, F., Gentile, F., De Angelis, F., Kumar, M. H. G., Candeloro, P., et al. (2009). Nano-patterned SERS substrate: Application for protein analysis vs. temperature. Biosensors and Bioelectronics, 24, 1693-9. 
8. Michaels, A. M., Jiang, J., \& Brus, L. (2000). Ag nanocrystal junctions as the site for surfaceenhanced Raman scattering of single rhodamine 6G molecules. The Journal of Physical Chemistry B, 104, 11965 .

9. Li, K., Stockman, M. I., \& Bergman, D. J. (2003). Self-similar chain of metal nanospheres as an efficient nanolens. Physical Review Letters, 91, 227402.

10. Dai, J., Čajko, F., Tsukerman, I., \& Stockman, M. (2008). Electrodynamic effects in plasmonic nanolenses. Physical Review Letters, 77, 115419.

11. Schofield, S. R., Studer, P., Hirjibehedin, C. F., Curson, N. J., Aeppli, G., \& Bowler, D. R. (2013). Quantum engineering at the silicon surface using dangling bonds. Nature Communications, 4, 1-7.

12. Coluccio, M. L., Gentile, F., Francardi, M., Perozziello, G., Malara, N., Candeloro, P., Di Fabrizio, E., et al. (2014). Electroless deposition and nanolithography can control the formation of materials at the nano-scale for plasmonic applications. Sensors, 14, 6056-6083.

13. Coluccio, M. L., Gentile, F., Das, G., Perozziello, G., Malara, N., Alrasheed, S., Candeloro, P., \& Di Fabrizio, E. (2015). From nucleotides to DNA analysis by a SERS substrate of a self similar chain of silver nanospheres. Journal of Optics, 17, 114021

14. Chirumamilla, M., Toma, A., Gopalakrishnan, A., Das, G., Zaccaria, R. P., Krahne, R., Rondanina, E., Leoncini, M., Liberale, C., De Angelis, F., \& Di Fabrizioe, E. (2014). 3D nanostar dimers with a sub-10-nm gap for single-/few-molecule surface-enhanced Raman scattering. Advanced Materials, 26, 2353-2358.

15. De Angelis, F., Proietti, R. Z., Francardi, M., Liberale, C., \& Di Fabrizio, E. (2011). Multischeme approach for efficient surface plasmon polariton generation in metallic conical tips on AFM-based cantilevers. Optics Express, 19, 22268-79.

16. Proietti, R. Z., Alabastri, A., De Angelis, F., Das, G., Liberale, C., \& Toma, A. (2012). Fully analytical description of adiabatic compression in dissipative polaritonic structures. Physical Review B, 86, 035410.

17. Proietti, R. Z., De Angelis, F., Toma, A., Razzari, L., Alabastri, A., \& Das, G. (2012). Surface plasmon polariton compression through radially and linearly polarized source. Optics Letters, $37,545-7$.

18. Coluccio, M. L., Francardi, M., Gentile, F., Candeloro, P., Ferrara, L., Perozziello, G., \& Di Fabrizio, E. (2016). Plasmonic 3D-structures based on silver decorated nanotips for biological sensing. Optics and Lasers in Engineering, 76(9), 45-51.

19. Coluccio, M. L., Gentile, F., Das, G., Nicastri, A., Perri, A. M., Candeloro, P., Perozziello, G., Proietti, R., Totero-Gongora, J. S., Alrasheed, S., Fratalocchi, A., Limongi, T., Cuda, G., \& Di Fabrizio, E. (2015). Detection of single amino acid mutation in human breast cancer by disordered plasmonic self-similar chain. Science Advances, 1(8), e1500487.

20. Kiyotsugu, Y., \& Yoshio, M. (2004). Role of BRCA1 and BRCA2 as regulators of DNA repair, transcription, and cell cycle in response to DNA damage. Cancer Science, 95, 866-871.

21. Scully, R., Ganesan, S., Vlasakova, K., Chen, J., Socolovsky, M., \& Livingston, D. M. (1999). Genetic analysis of BRCA1 function in a defined tumor cell line. Molecular Cell, 4, 10931099.

22. Prado, E., Colin, A., Servant, L., Lecomte, S., \& Phys, J. (2014). SERS spectra of oligonucleotides as fingerprints to detect label-free RNA in microfluidic devices. Journal Physical Chemistry C, 118, 13965-71.

23. Wu, Y. C., Lo, W. Y., Chiu, C. R., \& Yang, T. S. (2006). Surface enhanced Raman spectra of oligonucleotides induced by spermine. Journal of Raman Spectroscopy, 3, 799-807.

24. Perozziello, G., Møllenbach, J., Laursen, S., di Fabrizio, E., Gernaey, K., \& Krühne, U. (2012). Lab on a chip automates in vitro cell culturing. Microelectronic Engineering, 98, 655-658.

25. Whitesides, G. M. (2006). The origins and the future of microfluidics. Nature, 442(7101), 368373.

26. Simone, G., Perozziello, G., \& Nanosci, J. (2011). UV/Vis visible optical waveguides fabricated using organic-inorganic nanocomposite layers. Nanotechnology, 11(3), 2057-2063. 
27. Yu, A., Savas, T., Cabrini, S., Difabrizio, E., Smith, H. I., Stellacci, F., \& Am, J. (2005). High resolution printing of DNA feature on poly (methyl methacrylate) substrates using supramolecular nano-stamping. Chemical Society, 127(48), 16774-16775.

28. Zhang, Y., Park, S., Yang, S., \& Wang, T. H. (2010). An all-in-one microfluidic device for parallel DNA extraction and gene analysis. Biomedical Microdevices, 12(6), 1043-1049.

29. Keramas, G., Perozziello, G., Geschke, O., \& Christensen, C. B. V. (2004). Development of a multiplex microarray microsystem. Lab on a Chip, 4(2), 152-158.

30. Liu, K., \& Fan, Z. H. (1011). Thermoplastic microfluidic devices and their applications in protein and DNA analysis. Analyst (London), 136(7), 1288-1297.

31. Wu, J., Wu, X. Z., Huang, T., \& Pawliszyn, J. (2004). Analysis of proteins by CE, CIEF, and microfluidic devices with whole-column-imaging detection. In M. A. Strege, \& A. L. Lagu (Eds.), Methods in molecular biology, vol. 276: capillary electrophoresis of proteins and peptides (pp. 229-252). Totowa: Humana Press.

32. Perozziello, G., Candeloro, P., Gentile, F., Coluccio, M. L., Tallerico, M., De Grazia, A., Nicastri, A., Perri, A. M., Parrotta, E., Pardeo, F., Catalano, R., Cuda, G., \& Di Fabrizio, E. (2015). A microfluidic dialysis device for complex biological mixture SERS analysis. Microelectronic Engineering, 144, 37-41.

33. Tekin, H. C., Scherz, C., \& Gijs, M. A. M. (2011). Microfluidic device for analysis of protein biomarkers using magnetic bead surface coverage detection. In Proceedings of the 15th international conference on miniaturized systems for chemistry and life sciences (MicroTAS, 2011).

34. Perozziello, G., Candeloro, P., Gentile, F., Nicastri, A., Perri, A., Coluccio, M. L., Adamo, A., Pardeo, F., Catalano, R., Parrotta, E., Espinosa, H. D., Cuda, G., \& Di Fabrizio, E. (2014). Microfluidics \& nanotechnology: towards fully integrated analytical devices for the detection of cancer biomarkers. RSC Advances, 4(98), 55590-55598.

35. Perozziello, G., La Rocca, R., Cojoc, G., Liberale, C., Malara, N., Simone, G., Candeloro, P., Anichini, A., Tirinato, L., Gentile, F., Coluccio, M. L., Carbone, E., \& Di Fabrizio, E. (2012). Microfluidic devices modulate tumor cell line susceptibility to NK cell recognition, Small. Small, 8(18), 2886-2894.

36. Simone, G., \& Perozziello, G. (2010). $\mathrm{Ca}^{2+}$ mediates the adhesion of breast cancer cells in self-assembled multifunctional microfluidic chip prepared with carbohydrate beads. Micro and Nanosystems, 2(4), 261-268.

37. Simone, G., Perozziello, G., Battista, E., De Angelis, F., Candeloro, P., Gentile, F., Malara, N., Manz, A., Carbone, E., Netti, P., \& Di Fabrizio, E. (2012). Cell rolling and adhesion on surfaces in shear flow. A model for an antibody-based microfluidic screening system. Microelectronic Engineering, 98, 668-671.

38. Perozziello, G., Simone, G., Malara, N., La Rocca, R., Tallerico, R., Catalano, R., Pardeo, F., Candeloro, P., Cuda, G., Carbone, E., \& Di Fabrizio, E. (2013). Microfluidic biofunctionalisation protocols to form multi-valent interactions for cell rolling and phenotype modification investigations. Electrophoresis, 34(13), 1845-1851.

39. Perozziello, G., Catalano, R., Francardi, M., Rondanina, E., Pardeo, F., De Angelis, F., Malara, N., Candeloro, P., Morrone, G., \& Di Fabrizio, E. (2013). A microfluidic device integrating plasmonic nanodevices for Raman spectroscopy analysis on trapped single living cells. Microelectronic Engineering, 111, 314-319.

40. Kwan, J. M., Guo, Q., Kyluik-Price, D. L., Ma, H., \& Scott, M. D. (2013). Microfluidic analysis of cellular deformability of normal and oxidatively damaged red blood cells. American Journal of Hematology, 88(8), 682-689.

41. Faustino, V., Pinho, D., Yaginuma, T., Calhelha, R. C., Ferreira, I. C., \& Lima, R. (2014). Extensional flow-based microfluidic device: deformability assessment of red blood cells in contact with tumor cells. BioChip Journal, 8(1), 42-47.

42. Notingher, I., Verrier, S., Romanska, H., Bishop, A. E., Polak, J. M., \& Hench, L. L. (2002). In situ characterisation of living cells by Raman spectroscopy. Spectroscopy, 16, 43-51.

43. Verrier, S., Notingher, I., Polak, J. M., \& Hench, L. L. (2004). In situ monitoring of cell death using Raman microspectroscopy. Biopolymers, 74, 157-162. 
44. Uzunbajakava, N., Lenferink, A., Kraan, Y., Volokhina, E., Vrensen, G., Greve, J., \& Otto, C. (2003). Nonresonant confocal Raman imaging of DNA and protein distribution in apoptotic cells. Biophysical Journal, 84, 3968-3981.

45. Short, K. W., Carpenter, S., Freyer, J. P., \& Mourant, J. R. (2005). Raman spectroscopy detects biochemical changes due to proliferation in mammalian cell cultures. Biophysical Journal, 88 , 4274-4288.

46. Notingher, I., Jell, G., Lohbauer, U., Salih, V., \& Hench, L. L. (2004). In situ non-invasive spectral discrimination between bone cell phenotypes used in tissue engineering. Journal of Cellular Biochemistry, 92, 1180-1192.

47. Cheng, I. F., Chang, H.-C., Hou, D., \& Chang, H.-C. (2007). An integrated dielectrophoretic chip for continuous bioparticle filtering, focusing, sorting, trapping, and detecting. Biomicrofluidics, $1,21503$.

48. Huh, Y. S., Chung, A. J., \& Erickson, D. (2009). Surface enhanced Raman spectroscopy and its application to molecular and cellular analysis. Microfluidics and Nanofluidics, 6, 285-297.

49. Ramser, K., Enger, J., Goksoer, M., Hanstorp, D., Logg, K., \& Kaell, M. (2005). A microfluidic system enabling Raman measurements of the oxygenation cycle in single optically trapped red blood cells. Lab on a Chip, 5, 431-436.

50. Perozziello, G., Candeloro, P., De Grazia, A., Esposito, F., Allione, M., Coluccio, M. L., Tallerico, R., Valpapuram, I., Tirinato, L., Das, G., Giugni, A., Torre, B., Veltri, P., Kruhne, U., Della Valle, G., \& Di Fabrizio, E. (2016). Microfluidic device for continuous single cells analysis via Raman spectroscopy enhanced by integrated plasmonic nanodimers. Optics Express, 24(2), A180-A190.

51. Wood, B. R., Caspers, P., Puppels, G. J., Pandiancherri, S., \& McNaughton, D. (2007). Resonance Raman spectroscopy of red blood cells using near-infrared laser excitation. Analytical and Bioanalytical Chemistry, 387(5), 1691-1703.

52. Bankapur, A., Zachariah, E., Chidangil, S., Valiathan, M., \& Mathur, D. (2010). Raman tweezers spectroscopy of live, single red and white blood cells. PLoS One, 5(4), e10427.

53. Tallerico, R., Todaro, M., Di Franco, S., Maccalli, C., Garofalo, C., Sottile, R., Palmieri, C., Tirinato, L., Pangigadde, P. N., La Rocca, R., Mandelboim, O., Stassi, G., Di Fabrizio, E., Parmiani, G., Moretta, A., Dieli, F., Kärre, K., \& Carbone, E. (2013). Human NK cells selective targeting of colon cancer-initiating cells: A role for natural cytotoxicity receptors and MHC class I molecules. The Journal of Immunology, 190(5), 2381-2390.

54. Ong, Y. H., Lim, M., \& Liu, Q. (2012). Comparison of principal component analysis and biochemical component analysis in Raman spectroscopy for the discrimination of apoptosis and necrosis in K562 leukemia cells. Optics Express, 20(20), 22158-22171. 